\title{
The overlapping neurobiology of addiction and ADHD
}

\author{
Robert Eme* \\ Illinois School of Professional Psychology at Argosy University, Schaumburg Campus, USA
}

\begin{abstract}
Vulnerability to alcohol and drug addiction is due to a multitude of factors, including various mental disorders. This article examines how one such disorder, Attention-Deficit/Hyperactivity Disorder (ADHD), constitutes a vulnerability to addiction because of the overlapping neurobiology involving the mesolimbic and mesocortical dopamine neural networks.
\end{abstract}

\section{Introduction}

Alcohol and drug misuse and related substance use disorders, with addiction being the most severe form of substance use disorder, are a very serious problem in the United States, affecting millions and imposing enormous costs to the individual, family, and the community [1]. Since only a minority of people who use various substances become addicted, vulnerability to alcohol and drug addiction is due to a multitude of community, caregiver/family and individual risk factors including mental disorders [1-4]. One such mental disorder is Attention-Deficit/Hyperactivity Disorder (ADHD) [3]. The article will begin with a brief exposition of the previously reviewed research that establishes ADHD as a major risk for addiction [3]. It will then proceed to examine one of the most commonly accepted explanations for why mental disorders such ADHD increase risk for addiction, i.e., addiction and ADHD and addiction can be caused by deficits in shared, overlapping brain circuits $[1,2]$.

\section{ADHD as a major risk factor for addiction}

Two major research domains provide solid support for ADHD as a major risk factor for addiction. The first domain consists of numerous studies that have found a strikingly higher prevalence of ADHD among adolescents and young adults in treatment for addiction than would be expected from a population prevalence of between 5 and $7 \%$ for juveniles and between 3 and 5\% for adults [5]. For example, a large multisite National Institute of Health study of treatment of adolescents with substance use problems $(n=600)$ reported an ADHD prevalence of $38 \%$ for both boys and girls [6]. A meta-analysis of 29 international studies found an ADHD prevalence of $25.3 \%$ among adolescents and $21 \%$ among adults in treatment for substance use problems [7]. Lastly, and most recently, a 2016 study found a prevalence of ADHD between $34 \%$ and $46 \%$ in adult patients seeking treatment for cannabis use disorders [8].

The second domain consists of the many longitudinal studies that have found that childhood ADHD significantly increases risk for addiction in adolescents and beyond [3]. For example, a recent 2015 study examined the association between developmental trajectories of inattention, hyperactivity/impulsivity through childhood and adolescence (ages 8-16 years) and subsequent binge drinking and marijuana use in early adulthood (age 21 years) in the 579 child subjects in the landmark Multimodal Treatment Study of ADHD [9].
The results found that trajectories of worsening attention symptoms and delinquency (and less apparent improvement in hyperactivity/ impulsivity) were associated with higher rates of early adult binge drinking and marijuana use, compared with trajectories of stable or improving symptoms and delinquency.

In sum, research has convincingly shown that ADHD is an important risk factor for addiction. One reason for this increased risk is the overlapping neurobiology of addiction and ADHD.

\section{Neurobiology of addiction}

The two most important brain circuits in the neurobiology of addiction and ADHD involve the dopamine (DA) mesolimbic and mesocortical circuits [1,2]. In both of these circuits DA neurons ascend from the ventral tegmental area of the brain which is part of a group of structures located deep within the brain called the basal ganglia. In the mesolimbic pathway the critical connection is with the nucleus accumbens. It is this pathway that is most closely associated with motivation, incentive salience, and impulsivity. In the mesocortical pathway the critical connections are with prefrontal, orbital/frontal, and cingulate cortices. This pathway is associated with cognitive functions such as error detection, executive functions, and maintenance of goal directed behavior. In addition, this pathway exerts control over the mesolimbic DA system. These two neural circuits are closely connected in feedback/feedforward relationships such that disruptions in one circuit can adversely affect functioning in the other circuit.

The article will draw upon the findings of several current reviews that have shown that addiction to drugs and alcohol involves a repeating cycle of three stages with each stage linked to changes in the functioning of the mesolimbic and mesocortical circuits $[1,2,10,11]$.

The stages are: binge/intoxication, withdrawal/negative affect, and preoccupation/anticipation.

Correspondence to: Robert Eme, Illinois School of Professional Psychology at Argosy University, Schaumburg Campus, USA, Tel: 847-969-4949, E-mail: reme@argosy.edu

Key words: addiction, attention-deficit/hyperactivity disorder, dopamine

Received: December 16, 2016; Accepted: January 30, 2017; Published: February 03,2017 


\section{Stage 1: Binge/intoxication}

In this stage for many people initial substance use involves an element of impulsivity or sensation-seeking in which an individual consumes an intoxicating substance, experiences its rewarding or pleasurable consequences, and therefore makes the person more likely to take the substance again. This stage heavily involves the basal ganglia and the key sub-region of the nucleus accumbens. The pleasurable/ rewarding effects produced by addictive substances involve activity in the nucleus accumbens, including direct and indirect activation of the brain's dopamine system thereby causing sharp increases in the release of dopamine. Brain-imaging studies have documented that sharp, fast increases in dopamine triggered by addictive substances are associated with the 'high' accompanying drug abuse. Furthermore, the activation of the reward system not only generates pleasurable feelings but also initiates a conditioning process whereby stimuli present while using the substance can over time activate the dopamine system on their own and thereby trigger powerful urges/cravings termed incentive salience.' Thus, the increased dopamine signaling that follows exposure to the conditioned stimulus increases the likelihood that the individual will continue to be motivated to engage in the behaviors necessary to procure the substance of abuse.

\section{Stage 2: Withdrawal/negative affect}

In the withdrawal/negative affect stage, a person who has been using drugs or alcohol experiences withdrawal symptoms, which include negative emotions and sometimes symptoms of physical illness when they stop taking the substance. The negative feelings associated with withdrawal derive in large part from diminished activation in the reward circuitry of the basal ganglia. When used over the long-term all substances of abuse cause dysfunction in the brain's dopamine system through two mechanisms: a reduction in the number of dopamine (D2) receptors and a smaller release of dopamine than first experienced in the binge/intoxication stage. Collectively these two changes are referred to as a down-regulation of the dopamine system. As a consequence of this down-regulation, there is an overall reduction in the sensitivity of the brain's reward system both to addictive substances and to natural reinforcers such as food or sex which explains why those who develop an addiction often do not derive the same level of pleasure from oncepleasurable activities. This general loss of reward sensitivity triggers future rounds of binge/intoxication and compulsive escalation of substance use as addicted individuals attempt to regain the pleasurable feelings the reward system once provided.

\section{Stage 3: Preoccupation/anticipation stage}

In the preoccupation/anticipation stage, a person begins to seek/crave substances again after periods of abstinence that can be quite short, i.e., a couple of hours. This stage of addiction involves a compromise in the functioning of the brain's Prefrontal Cortex (PFC) which is the region that mediates executive function (EF). Although definitions of EF vary considerably, a generally accepted broad definition of $\mathrm{EF}$ is that it refers to cognitive processes that are required for top-down control of behavior, emotions and thoughts, involving the ability to organize thoughts and activities, prioritize tasks, manage time, make decisions [12]. Thus EF is essential for a person to make appropriate choices about whether or not to use a substance and to resist strong urges to use. In this third stage, these EF abilities become impaired through a disruption in mesocortical DA system which exerts control of the subcortical mesolimbic DA system. Since there are dopaminergic projections to the PFC from the midbrain DA system, as well as DA neurons in the PFC itself [2], the same down-regulation of the dopamine system that blunted the reward circuit's sensitivity to pleasure also occurs in the PFC and thereby disrupts its functioning.

In sum, the concurrent down-regulation of both the mesolimbic and mesocortical DA systems simultaneously increases craving for addictive substances and reduces the ability to control such craving.

\section{Neurobiology of ADHD}

In discussing the neurobiology of $\mathrm{ADHD}$, the article will draw on several current, authoritative reviews of the neurobiology of ADHD and especially on the work of Theodore Beauchaine and colleagues as they have presented the most comprehensive discussions explaining why ADHD is a risk factor for addiction, i.e., ADHD and addiction can both be caused by shared, overlapping neurobiological deficits in the mesolimbic and mesocortical DA systems [2,13-18]. As most recently articulated, "Externalizing spectrum disorders including ADHD....and substance use disorder, share genetic, temperamental, subcortical, and cortical vulnerabilities" [18]. These shared neurobiological cortical and subcortical vulnerabilities will now be discussed in terms of the three core psychological dysfunctions in the addiction cycle: impulsivity, diminished sensitivity to reward, and impaired control.

\section{Stage 1: Impulsivity}

As previously discussed, for many people initial substance use involves an element of impulsivity or sensation-seeking in which an individual consumes an intoxicating substance. Since the core features of ADHD involve the deficient control of behavior and emotion resulting in behavioral and emotional impulsivity [19], ADHD is widely conceptualized as a disorder of self-regulation [14,16,19]. Indeed, ADHD has been proclaimed to be "paradigmatic of problems in the domain of self-regulation" [14].Thus ADHD with its severe impairments in self-control and can markedly increase the risk for initial substance use either directly because of behavioral/emotional impulsivity or indirectly as the first stage in a developmental pathway in which a sizable proportion of children with ADHD can progress to increasingly severe forms of antisocial behavior thereby increasing the risk for addiction [15-17].

\section{Stage 2: Diminished sensitivity to reward: mesolimbic DA system}

Contemporary neural models of ADHD impulsivity are strikingly similar to the neurobiology of addiction in that they focus primarily on dysfunctions in the mesolimbic and mesocortcal DA systems that cause the same deficits of impaired sensitivity to reward and impaired control that characterize stages 2 and 3 in the addiction cycle. With regard to impaired sensitivity to reward, extensive research has established that ADHD is caused in part by deficient mesolimbic DA activity, i.e., chronically low tonic DA and diminished DA phasic reactivity to rewards [13-18]. This deficient activity produces, as with addiction, a blunted response to typical rewards that is experienced in part as an "intolerance for boredom" [20]. This aversive state in turn provides motivation to engage in impulsive reward seeking, noveltyseeking behaviors that will activate the neurons in the mesolimbic DA system. Thus adolescents who are prone to risky substance use "may seek the more extreme reward of drugs because their experience of conventional rewards is blunted" [21]. However, since these behaviors do not address the underlying DA deficiency, they produce only temporary improvements in mood and thus larger and more frequent rewards are sought. 


\section{Stage 3: Impaired control: mesocortical DA system}

There is virtual unanimity that ADHD is caused in part by deficits in EF functioning which contribute to behavioral and emotional impulsivity $[13,14,19]$. Furthermore, a voluminous literature has found that a dysfunctional mesocortical DA system is implicated in ADHD impulsivity $[13,14,16-19]$. This dysfunction, as in addiction, produces a deficient regulation of the impulsive reward-seeking behavior caused by the impaired mesolimbic DA system. In sum, a compromised subcortical mesolimbic DA system and a compromised mesocortical DA system contributed to impulsive reward-seeking, novelty-seeking behaviors that markedly increase risk for addiction.

\section{Conclusion}

The remarkable parallel between the three major deficits in psychological functioning in addiction and ADHD of impulsivity, diminished sensitivity to reward, and impaired control can be explained by the overlapping neurobiological deficits in both disorders that undergird the common psychological deficits. These deficits involve dysfunctions in the mesolimbic and mesocortical DA systems which in ADHD are neurodevelopment in origin and in addiction are acquired. Since it is precisely these acquired deficits in the mesolimbic and mesocortical DA systems that propel the three stage addiction cycle, it can readily be seen how these same neurodevelopmental deficits in ADHD can help initiate such a cycle, and therefore why ADHD is a major risk factor for addiction.

\section{References}

1. U.S. Department of Health and Human Services, Office of the Surgeon General (2016). Facing addiction is America: The Surgeon General's report on alcohol, drugs, and health. Washington DC.

2. Patrick C, Foeli J, Venables N, Worthy (2016) Substance use disorders and externalizing outcomes. In. T. Beauchaine \& S. Hinshaw (Eds.), The Oxford handbook of externalizing spectrum disorders (38-60). New York: Oxford University Press.

3. Eme R (2016) ADHD and risky substance abuse in male adolescents. The ADHD Report 24: 1-8.

4. Abram KM (2016) New Evidence for the Role of Mental Disorders in the Development of Substance Abuse. J Am Acad Child Adolesc Psychiatry 55: 265-266. [Crossref]

5. Roberts W, Milich R, Barkley R (2015) Primary symptoms, diagnostic criteria, subtyping, and prevalence of ADHD. In R. Barkley (Ed.), Attention-deficit hyperactivity disorder (4th ed., pp. 50-80). New York: Guilford
6. Dennis M, Godley SH, Diamond G, Tims FM, Babor T, et al. (2004) The Cannabis Youth Treatment (CYT) Study: main findings from two randomized trials. J Subst Abuse Treat 27: 197-213. [Crossref]

7. van Emmerik-van Oortmerssen K, van de Glind G, van den Brink W, Smit F, Crunelle CL, et al. (2012) Prevalence of attention-deficit hyperactivity disorder in substance use disorder patients: a meta-analysis and meta-regression analysis. Drug Alcohol Depend 122: 11-19. [Crossref]

8. Notzon D, Pavlicova M, Glass A, Mariani J, Mahony A, et al. (2016) ADHD is highly prevalent in patients seeking treatment for cannabis use disorders. $J$ Atten Dis. [Crossref]

9. Howard AL, Molina BS, Swanson JM, Hinshaw SP, Belendiuk KA, et al. (2015) Developmental progression to early adult binge drinking and marijuana use from worsening versus stable trajectories of adolescent attention deficit/hyperactivity disorder and delinquency. Addiction 110: 784-795. [Crossref]

10. Volkow ND, Koob GF, McLellan AT (2016) Neurobiologic Advances from the Brain Disease Model of Addiction. N Engl J Med 374: 363-371. [Crossref]

11. Koob GF, Volkow ND2 (2016) Neurobiology of addiction: a neurocircuitry analysis. Lancet Psychiatry 3: 760-773. [Crossref]

12. Diamond A (2013) Executive functions. Annu Rev Psychol 64: 135-168. [Crossref]

13. Faraone S, Asherson P, Banaschewski T, Biederman J, Buitelaar J, et al. (2015) Attention-deficit/hyperactivity disorder. Nature Reviews: Disease Primers 1: 1-23.

14. Nigg J (2016) Attention and impulsivity. In D. Cicchetti (Ed.). Developmental psychopathology: Risk, disorder and adaptation (3rd ed.). New York: Wiley. 3: 591-646

15. Beauchaine T, McNulty T (2013) Comorbidities and continuities as ontogenic processes: towards a developmental spectrum model of externalizing psychopathology. Dev Psychopathol 25: 1505-1527. [Crossref]

16. Zisner A, Beauchaine TP (2016) Neural substrates of trait impulsivity, anhedonia, and irritability: mechanisms of heterotypic comorbidity between externalizing disorders and unipolar depression. Dev Psychopathol 28: 1179-1210. [Crossref]

17. Beauchaine T, Zisner A, Sauder C (in press). Trait impulsivity and the externalizing spectrum. Annual review of clinical psychology.

18. Beauchaine TP, Cicchetti D (2016) A new generation of comorbidity research in the era of neuroscience and Research Domain Criteria. Dev Psychopathol 28: 891-894. [Crossref]

19. Barkley R (2015) Attention-Deficit Hyperactivity Disorder: a handbook for diagnosis and treatment (4th ed.): New York: Guilford Press.

20. Schneider S, Peters J, Bromberg U, Brassen S, Miedl SF, et al. (2012) Risk taking and the adolescent reward system: a potential common link to substance abuse. Am J Psychiatry 169: 39-46. [Crossref]

21. Schoenfelder EN, Faraone SV, Kollins SH (2014) Stimulant treatment of ADHD and cigarette smoking: a meta-analysis. Pediatrics 133: 1070-1080. [Crossref]

Copyright: (C2017 Eme R. This is an open-access article distributed under the terms of the Creative Commons Attribution License, which permits unrestricted use, distribution, and reproduction in any medium, provided the original author and source are credited. 